\title{
Inmunoterapia intralinfática para el manejo de patologías alérgicas en el paciente pediátrico. Revisión sistemática de ensayos clínicos
}

\author{
Intralymphatic immunotherapy for the management of allergic pathologies \\ in the pediatric patient. Systematic review of clinical trials
}

\author{
Dra. Blanca Lilia Martínez Olivier, ${ }^{\star}$ Dr. Álvaro Pedroza Meléndez, ${ }^{\ddagger}$ \\ Dr. Francisco Alberto Contreras Verduzco ${ }^{\ddagger}$ \\ * Especialidad en Alergia e Inmunología Clínica Pediátrica. \\ ₹ Médico adscrito al Área de Alergia Pediátrica. \\ Instituto Nacional de Pediatría. CDMX, México.
}

Citar como: Martínez OBL, Pedroza MÁ, Contreras VFA. Inmunoterapia intralinfática para el manejo de patologías alérgicas en el paciente pediátrico. Revisión sistemática de ensayos clínicos. Alergia Asma Inmunol Pediatr. 2021; 30 (1): 5-12. https://dx.doi.org/10.35366/100111

\section{RESUMEN}

Introducción: La inmunoterapia intralinfática (IIL) ha ganado interés tanto por su fácil abordaje como por las bondades potenciales, que incluyen una presentación antigénica efectiva a células residentes del ganglio linfático. Sin embargo, la evidencia actual es escasa y no existen revisiones sistemáticas de ensayos clínicos que permitan conocer la evidencia sobre su eficacia. Objetivo: Realizar una revisión sistemática de ensayos clínicos sobre la utilidad de la inmunoterapia intralinfática para el manejo de enfermedades alérgicas pediátricas. Material y métodos: Se realizó una búsqueda en PubMed, EBSCO e ISI Web of Knowledge con los siguientes términos, sin límite de tiempo ni idioma: intralymphatic, immunotherapy, allergy. Se seleccionaron ensayos clínicos de IIL para enfermedades alérgicas en estudios realizados exclusivamente o que incluyeran a pacientes de 18 años o menores. Se siguieron los criterios PRISMA para revisiones sistemáticas. El riesgo de sesgo se evaluó con la herramienta Cochrane. Resultados: De 274 registros se incluyeron cinco estudios que sumaron 73 individuos que recibieron placebo y 79 pacientes que recibieron al menos tres dosis de 1,000 SQ-U de

\section{ABSTRACT}

Introduction: Intralymphatic immunotherapy (IIL) has gained interest because of its easy approach as well as potential benefits, which include effective antigenic presentation to resident lymph node cells. However, current evidence is scarce and there are no systemic reviews of clinical trials to provide evidence of its efficacy. Objective: To conduct a systematic review of clinical trials on the usefulness of intralymphatic immunotherapy for the management of pediatric allergic diseases. Material and methods: $A$ search was carried out in PubMed, EBSCO and ISI Web of Knowledge with the following terms, with no time or language limit: intralymphatic immunotherapy allergy. Clinical trials of IIL for allergic diseases in studies conducted exclusively or involving patients 18 years of age or younger were selected. PRISMA criteria for systematic reviews were followed. Risk of bias was assessed using the Cochrane tool. Results: Of 274 records, five studies were included, totaling 73 individuals who received placebo and 79 patients who received at least 3 doses of 1,000 SQ-U of IIL with a follow-up of 10-52 weeks. In patients with rhinitis and allergic rhinoconjunctivitis, IIL improved the nasal challenge test and decreased

Abreviaturas:

IIL = Inmunoterapia intralinfática. IEA = Inmunoterapia específica para alérgenos. $\mathrm{RA}=$ Rinitis alérgica. $\mathrm{RC}=$ Rinoconjuntivitis. $\mathrm{RCyAL}=$ Rinoconjuntivitis y asma leve.

Recibido: 09/03/2021. Aceptado: 17/03/2021.

Correspondencia: Blanca Lilia Martínez Olivier

Insurgentes Sur 3700 Letra C, Insurgentes Cuicuilco, 04530, Alcaldía Coyoacán, Ciudad de México. Teléfono: 23 8142-7284

E-mail: dra.blancaolivier@gmail.com 
IIL con un seguimiento de 10-52 semanas. En pacientes con rinitis y rinoconjuntivitis alérgica la IIL mejoró la prueba de provocación nasal y disminuyó los síntomas locales y sistémicos. Además, condujo a una reducción del consumo de fármacos y a la mejoría de parámetros inmunológicos en la mayoría de los estudios. La IIL no produjo eventos adversos moderados a severos, sólo leves que incluyeron: comezón, urticaria, inflamación en el sitio de inyección, estornudos y cansancio. La adherencia terapéutica fue cercana a $100 \%$. En pacientes con asma leve la evidencia es insuficiente. Conclusiones: La IIL es una estrategia terapéutica efectiva y segura para el tratamiento de rinitis y rinoconjuntivitis alérgica que se asocia con una excelente adherencia y requiere sólo de tres dosis para tener un efecto terapéutico.

Palabras clave: Alergia, inmunoterapia intralinfática, eficacia, eventos adversos. local and systemic symptoms. In addition, it led to a decrease in drug consumption and improvement of immunological parameters in most studies. IIL did not produce moderate to severe adverse events, only mild ones; including: itching, urticaria, inflammation at the injection site, sneezing and tiredness. Adherence to therapy was close to $100 \%$. In patients with mild asthma the evidence is insufficient. Conclusions: IIL is an effective and safe therapeutic strategy for the treatment of allergic rhinitis and rhinoconjunctivitis that is associated with excellent adherence and requires only 3 doses to have a therapeutic effect.

Keywords: Allergy, intralymphatic immunotherapy, efficacy, adverse events.

\section{INTRODUCCIÓN}

La inmunoterapia específica para alérgenos (IEA) es el único tratamiento de alergia que confiere una mejoría de los síntomas a largo plazo. ${ }^{1}$ La ruta de aplicación de alérgenos más utilizada es la inyección subcutánea, comúnmente tomada como el estándar de oro, pero otras vías como la aplicación sublingual, epicutánea e intralinfática (IIL) se encuentran en desarrollo clínico. ${ }^{2}$ La IEA modifica la respuesta inmune a través de la exposición repetida a largo plazo a dosis definidas de alérgenos; induce tolerancia periférica activando células T reguladoras (Tregs) y en consecuencia suprime diferentes células proinflamatorias, como eosinófilos, mastocitos, basófilos y células B, modulando la producción de citocinas pro y antiinflamatorias, así como de anticuerpos..$^{3,4}$

La aplicación de la IIL no es dolorosa y es segura, ya que se aplica directamente en el ganglio linfático con apoyo del ultrasonograma y se han reportado buenos resultados con sólo tres dosis bajas con un mes de diferencia, ${ }^{5,6}$ pero otros autores sugieren que se requiere un tratamiento por varios años para que sea efectivo. ${ }^{7}$

Entre las ventajas de la IIL sobre otros tipos de IEA se encuentran que al aplicarse directo en los nodos linfáticos se requieren menos dosis y tras una sola de éstas puede distribuirse de manera eficiente en hasta cinco nódulos linfáticos, mientras que una pequeña cantidad de la aplicación subcutánea llega a los ganglios. ${ }^{8}$ Asimismo, la IIL induce respuestas de IgG 10 veces más altas que la subcutánea con dosis de alérgenos 100 veces menores, lo cual, potencialmente, podría conllevar a menos efectos adversos. ${ }^{9}$ Además, su aplicación en tres dosis podría contribuir a una mejor adherencia, evitar interrupciones y terminaciones prematuras del tratamiento. ${ }^{8,9}$ En contraste, la IEA subcutánea requiere de 30-80 inyecciones durante 3-5 años. ${ }^{10}$
Aunque, de manera reciente, se han realizado diversos ensayos clínicos sobre la efectividad de la IIL en comparación con placebo o con IEA, la mayoría han incluido sólo a pacientes adultos, por lo que se conoce muy poco sobre su uso en población pediátrica, pero podría ser igual de promisorio que en el adulto. ${ }^{11}$ En el presente estudio se muestra una revisión sistemática de ensayos clínicos sobre la utilidad de la inmunoterapia intralinfática (IIL) para el manejo de enfermedades alérgicas pediátricas.

\section{MATERIAL Y MÉTODOS}

\section{Criterios de inclusión de artículos}

Se buscaron artículos originales sobre la utilidad de la IIL para el manejo de enfermedades alérgicas pediátricas. Dado que no se encontraron artículos realizados exclusivamente en pacientes pediátricos, se seleccionaron los estudios originales tipo ensayos clínicos que incluyeran a pacientes menores de 20 años en quienes se aplicara IIL y se comparara con un grupo placebo; por lo que los estudios debieron incluir al menos dos grupos (uno con terapia IIL y otro control) y reportar información relacionada con las características del tratamiento, el tiempo de seguimiento y los desenlaces.

\section{Estrategia de búsqueda y fuentes de información}

Se realizó una búsqueda en PubMed, Web of Knowledge y Scopus con los siguientes términos, sin límite de tiempo ni idioma: («intralymphatic» [All Fields] OR «intralymphatically» [All Fields]) AND ((«immunotherapy» [MeSH Terms] OR «immunotherapy» [All Fields]) OR «immunotherapies» [All Fields]) AND (((c((«allergie» [All Fields] OR «hypersensitivity» [MeSH Terms]) OR «hypersensitivity» [All Fields]) OR «allergies» [All Fields]) OR «allergy» [All Fields]) OR «al- 


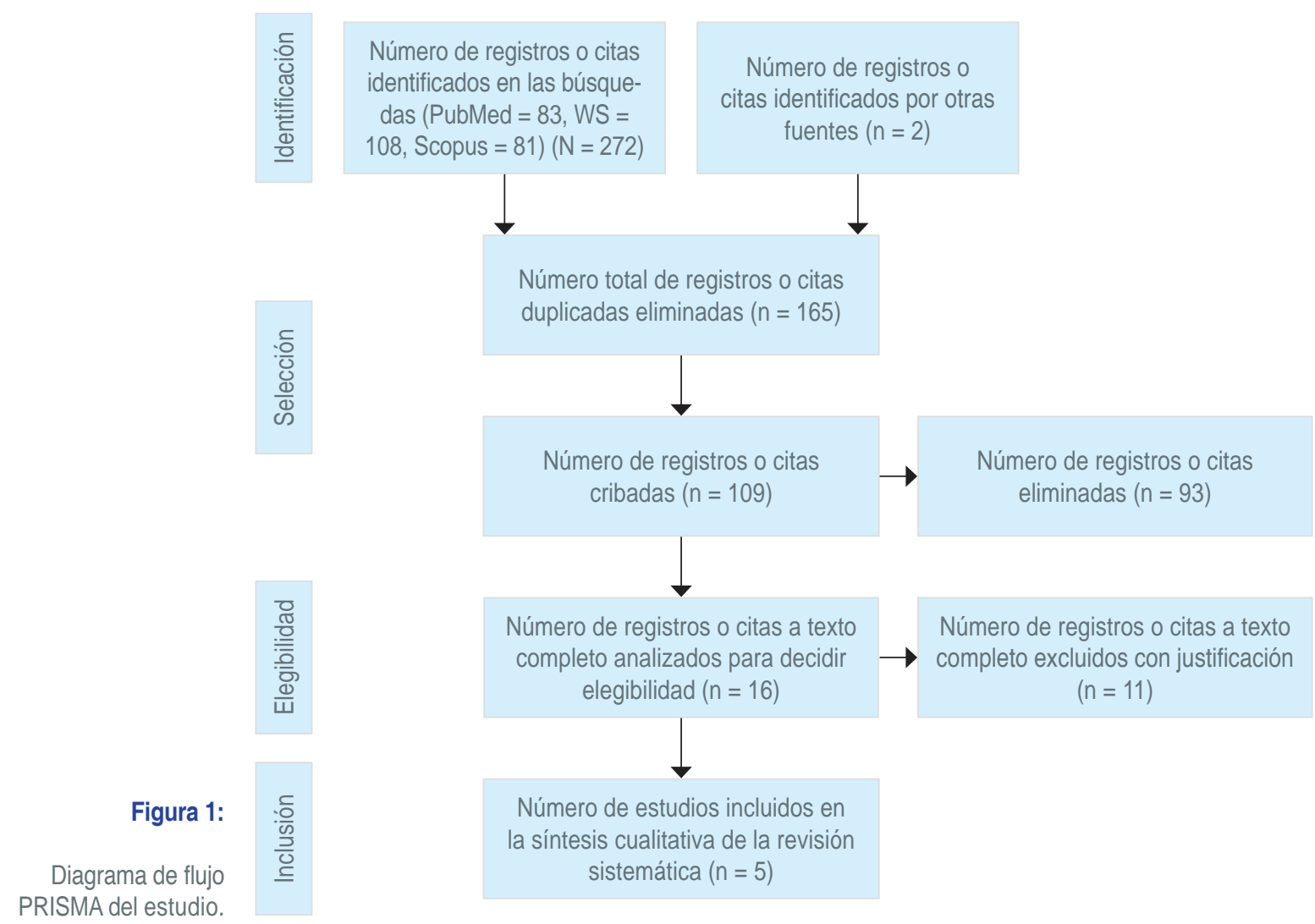

lergy and immunology» [MeSH Terms]) OR («allergy» [All Fields] AND «immunology» [All Fields])) OR «allergy and immunology» [All Fields]). Manualmente se buscaron en publicaciones relevantes estudios adicionales que fueran potencialmente relevantes.

\section{Selección de estudios y extracción de datos}

Para la realización del estudio se utilizaron los criterios PRIS$\mathrm{MA}^{12,13}$ para revisiones sistemáticas. Todos los resúmenes encontrados sobre el tópico de interés fueron valorados por dos evaluadores independientes y en caso de discrepancia en la selección, un tercer investigador resolvió de forma independiente y cegada el desacuerdo. Aquellos estudios que cumplieron los criterios de inclusión fueron revisados a texto completo para identificar los estudios que sí reportaran los hallazgos de interés. Se extrajo la siguiente información de las publicaciones: autor, año, país, patología alérgica, grupos, tamaño de muestra por grupo y total, edad y sexo de los participantes, alérgenos utilizados, dosis del alérgeno por aplicación, total de aplicaciones, el comparador (placebo), sitio de aplicación de inmunoterapia, tiempo de seguimiento, desenlaces medidos, resultados clínicos y eventos adversos.

\section{Evaluación del riesgo de sesgo}

El riesgo de sesgo de los artículos seleccionados fue evaluado por dos revisores (B.O. y R.V.) de forma independiente. La evaluación de la calidad se realizó mediante el software Cochrane RevMan v. 5.3. Cada artículo se evaluó en las siguientes dimensiones: sesgo de selección, sesgo de ejecución, sesgo de detección, sesgo de deserción, sesgo de notificación y otros sesgos, de acuerdo con las recomendaciones de Cochrane. ${ }^{14}$

\section{RESULTADOS}

\section{Estudios seleccionados}

Con los criterios de búsqueda establecidos se identificaron un total de 274 registros, tras eliminar duplicados (165 estudios) se revisaron 109 resúmenes para identificar artículos originales tipo ensayo clínico que evaluaran la efectividad de la IIL para el manejo de enfermedades alérgicas en menores de 20 años. De éstos, 11 artículos fueron revisados a texto completo para decidir elegibilidad y en la síntesis cualitativa (revisión sistemática) se incluyeron un total de cinco estudios (Figura 1). 
Tabla 1: Características generales de los estudios incluidos.

\begin{tabular}{|c|c|c|c|c|c|c|c|c|c|c|}
\hline Autor, año & País & $\begin{array}{l}\text { Tipo de } \\
\text { estudio }\end{array}$ & $\begin{array}{l}\text { Patología } \\
\text { alérgica }\end{array}$ & $\begin{array}{c}\text { Tamaño } \\
\text { de } \\
\text { muestra (n) }\end{array}$ & $\begin{array}{l}\text { Edad } \\
\text { (años) }\end{array}$ & $\begin{array}{l}\text { Sexo } \\
(\mathrm{M} / \mathrm{F})\end{array}$ & $\begin{array}{l}\text { Número } \\
\text { y tipo de } \\
\text { alérgenos } \\
\text { utilizados }\end{array}$ & $\begin{array}{l}\text { Compara- } \\
\text { dor/placebo }\end{array}$ & $\begin{array}{l}\text { Posología del } \\
\text { alérgeno* }\end{array}$ & $\begin{array}{l}\text { Tiempo de } \\
\text { seguimiento } \\
\text { (semanas) }\end{array}$ \\
\hline $\begin{array}{l}\text { Konradsen, } \\
2020\end{array}$ & Suecia & $\begin{array}{l}\text { Ensayo clí- } \\
\text { nico, doble } \\
\text { ciego, alea- } \\
\text { torizado, } \\
\text { controlado } \\
\text { con pla- } \\
\text { cebo }\end{array}$ & $\begin{array}{l}\text { Rinocon- } \\
\text { juntivitis y } \\
\text { asma leve }\end{array}$ & $\begin{array}{l}\text { Total 26; } \\
\text { Grupo IIL14 } \\
\text { Grupo con- } \\
\text { trol } 12\end{array}$ & $16-42$ & $\begin{array}{l}\text { 6/8 (grupo } \\
\text { inmuno- } \\
\text { terapia); } \\
10 / 2 \\
\text { (grupo } \\
\text { placebo) }\end{array}$ & $\begin{array}{l}\text { 2: extracto } \\
\text { de abedul } \\
\text { y polen } \\
\text { de hierba } \\
\text { (pasto) }\end{array}$ & Placebo & $\begin{array}{l}1,000 \mathrm{SQ}-U, \\
3 \text { aplicaciones } \\
\text { previo a la } \\
\text { primera tempo- } \\
\text { rada de polen } \\
\text { y una antes } \\
\text { de la segunda } \\
\text { temporada, } \\
\text { separadas por } \\
\text { un mes }\end{array}$ & 52 \\
\hline $\begin{array}{l}\text { Hellkvist, } \\
2018\end{array}$ & Suecia & $\begin{array}{l}\text { Ensayo clí- } \\
\text { nico, doble } \\
\text { ciego, alea- } \\
\text { torizado, } \\
\text { controlado } \\
\text { con pla- } \\
\text { cebo }\end{array}$ & $\begin{array}{l}\text { Rinitis } \\
\text { alérgica }\end{array}$ & $\begin{array}{l}\text { Total 60; } \\
\text { Grup o IIL30 } \\
\text { Grupo con- } \\
\text { trol } 30\end{array}$ & $18-55$ & $\begin{array}{l}18 / 6 \\
\text { (grupo ex- } \\
\text { perimen- } \\
\text { tal); } 27 / 10 \\
\text { (grupo } \\
\text { placebo) }\end{array}$ & $\begin{array}{l}\text { 2: extracto } \\
\text { de abedul } \\
\text { y polen } \\
\text { de hierba } \\
\text { (pasto) }\end{array}$ & $\begin{array}{l}\text { Doble } \\
\text { placebo } \\
\text { (diluyente } \\
\text { ALK) }\end{array}$ & $\begin{array}{l}1,000 \mathrm{SQ}-U, 3 \\
\text { aplicaciones to- } \\
\text { tales separadas } \\
\text { por un mes }\end{array}$ & 42 \\
\hline $\begin{array}{l}\text { Hylander, } \\
2016\end{array}$ & Suecia & $\begin{array}{l}\text { Ensayo clí- } \\
\text { nico, doble } \\
\text { ciego, alea- } \\
\text { torizado, } \\
\text { controlado } \\
\text { con pla- } \\
\text { cebo }\end{array}$ & $\begin{array}{l}\text { Rinocon- } \\
\text { juntivitis } \\
\text { alérgica }\end{array}$ & $\begin{array}{l}\text { Total 36; } \\
\text { Grupo IIL21 } \\
\text { Grupo con- } \\
\text { trol } 15\end{array}$ & $18-65$ & $\begin{array}{l}13 / 8 \\
\text { (grupo } \\
\text { activo); } \\
9 / 6 \text { (grupo } \\
\text { control) }\end{array}$ & $\begin{array}{l}\text { 2: extracto } \\
\text { de abedul } \\
\text { y polen } \\
\text { de hierba } \\
\text { (pasto) }\end{array}$ & $\begin{array}{l}\text { Placebo } \\
\text { (diluyente } \\
\text { ALK) }\end{array}$ & $\begin{array}{l}1,000 \mathrm{SQ}-\mathrm{U}, 3 \\
\text { aplicaciones to- } \\
\text { tales separadas } \\
\text { por un mes }\end{array}$ & 24 \\
\hline $\begin{array}{l}\text { Patterson, } \\
2016\end{array}$ & EUA & $\begin{array}{l}\text { Ensayo clí- } \\
\text { nico, doble } \\
\text { ciego, alea- } \\
\text { torizado, } \\
\text { controlado } \\
\text { con pla- } \\
\text { cebo }\end{array}$ & $\begin{array}{l}\text { Rinitis } \\
\text { alérgica }\end{array}$ & $\begin{array}{l}\text { Total } 15 \\
\text { Grupo IIL7 } \\
\text { Grupo con- } \\
\text { trol } 8\end{array}$ & $15-24$ & $\begin{array}{l}\text { No repor- } \\
\text { tado }\end{array}$ & $\begin{array}{l}\text { 1: extracto } \\
\text { de polen } \\
\text { de hierba } \\
\text { (pasto) }\end{array}$ & $\begin{array}{l}\text { Placebo } \\
\text { (solución } \\
\text { salina } \\
\text { normal con } \\
\text { fenol) }\end{array}$ & $\begin{array}{l}20,000 \text { PNU/ } \\
\text { mL, } 3 \text { aplica- } \\
\text { ciones totales } \\
\text { separadas por } \\
\text { un mes }\end{array}$ & 10 \\
\hline $\begin{array}{l}\text { Hylander, } \\
2013\end{array}$ & Suecia & $\begin{array}{l}\text { Ensayo clí- } \\
\text { nico, doble } \\
\text { ciego, alea- } \\
\text { torizado, } \\
\text { controlado } \\
\text { con pla- } \\
\text { cebo }\end{array}$ & $\begin{array}{l}\text { Rinitis } \\
\text { alérgica }\end{array}$ & $\begin{array}{l}\text { Total 15; } \\
\text { Grupo IIL } 7 \\
\text { Grupo con- } \\
\text { trol } 8\end{array}$ & $19-53$ & $\begin{array}{l}4 / 3 \text { (grupo } \\
\text { experi- } \\
\text { mental); } \\
6 / 2 \text { (grupo } \\
\text { placebo) }\end{array}$ & $\begin{array}{l}\text { 2: extracto } \\
\text { de abedul } \\
\text { y polen } \\
\text { de hierba } \\
\text { (pasto) }\end{array}$ & $\begin{array}{l}\text { Placebo } \\
\text { (diluyente } \\
\text { ALK) }\end{array}$ & $\begin{array}{l}1,000 \mathrm{SQ}-U, 3 \\
\text { aplicaciones to- } \\
\text { tales separadas } \\
\text { por un mes }\end{array}$ & 24 \\
\hline
\end{tabular}

* El sitio de aplicación en todos los casos fueron los ganglios linfáticos inguinales. 


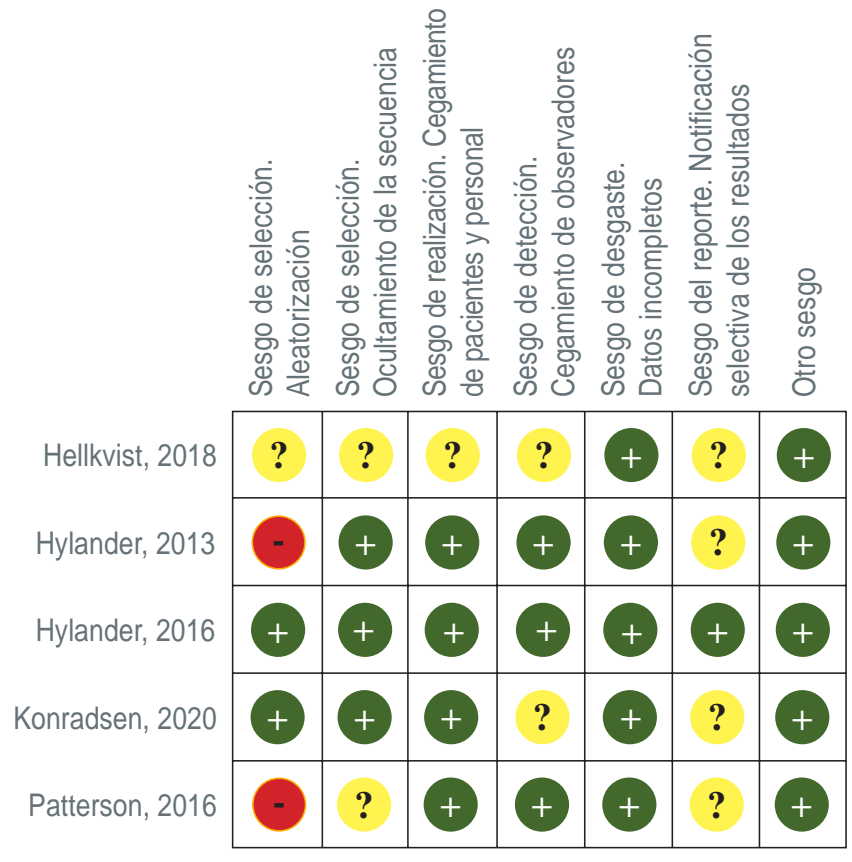

Figura 2: Síntesis de riesgo de sesgo de cada estudio incluido.

\section{Características de los estudios y pacientes incluidos}

Los estudios incluidos ${ }^{15-19}$ fueron realizados entre 2013 y 2020 en Suecia $(n=4)$ y Estados Unidos de América $(n=$ 1). Tres estudios fueron realizados en pacientes con rinitis alérgica, ${ }^{15,16,18}$ uno en rinoconjuntivitis alérgica ${ }^{17}$ y otro en rinoconjuntivitis y asma leve. ${ }^{19}$ Todos los estudios fueron comparaciones de IIL con placebo. ${ }^{15-19}$ Las dosis empleadas de alérgenos fueron tres aplicaciones de 1,000 SQ-U del alérgeno con separación de un mes entre aplicaciones en tres estudios, en otro la misma dosis pero cuatro aplicaciones totales y en el último un total de tres aplicaciones a una dosis de 20,000 PNU/mL, con una separación de un mes entre aplicación (Tabla 1). El total de individuos incluidos en los grupos control fueron 73 pacientes y en los grupos de intervención fueron 79 pacientes. Dado que no se encontraron estudios realizados exclusivamente en pacientes pediátricos, se seleccionaron los estudios que incluyeran pacientes $\leq 18$ años. Las edades de los pacientes de los estudios incluidos oscilaron entre 16 y 55 años (Tabla 1).

\section{Riesgo de sesgo}

La valoración del riesgo de sesgo con la herramienta Cochrane arrojó los siguientes resultados: en un estudio el riesgo de sesgo fue claro para la mayoría de los criterios, ${ }^{18}$ se encontró riesgo de selección en dos estudios, pero la mayoría de los criterios restantes fueron evaluados como bajo riesgo de sesgo (Figura 2). Por lo que, en general, los estudios fueron de buena calidad con baja probabilidad de sesgo de ejecución, de detección, de deserción, de notificación y otros sesgos, de acuerdo con los criterios de Cochrane (Figura 3). ${ }^{14}$

\section{Desenlaces evaluados y resultados principales}

Los principales parámetros evaluados en los estudios incluidos fueron: síntomas de alergia estacional, uso de medicamentos, prueba de Prick en piel, prueba de provocación nasal, mediciones en líquido de lavado nasal, parámetros inmunológicos y eventos adversos. En otros estudios se evaluaron algunos parámetros compuestos como el puntaje de síntomas locales y sistémicos (total safety score [TSS]) y el puntaje de síntomas y uso de medicamentos para alergia y asma (total combined score [TCS]). En la Tabla 2 se simplifican los principales hallazgos de los estudios incluidos en términos de mejoría, empeoramiento o ausencia de cambios.

En pacientes con rinitis alérgica (RA) [tres estudios incluidos] la IIL indujo mejoras en la prueba de provocación nasal en dos estudios ${ }^{15,18}$ y en uno no se especificó. ${ }^{16}$ Todos los estudios reportaron mejoría de síntomas locales y sistémicos, así como disminución del uso de medicamentos con la aplicación de la IIL. En dos de tres estudios se reportó mejoría en parámetros inmunológicos objetivados mediante disminución en el total de leucocitos e IL-8 en fluido nasal, aumento de la expresión de CD69 y CD98 en linfocitos CD4+ en sangre periférica ${ }^{15}$ y mediante disminución del tamaño de la reacción en la prueba de Prick en piel. ${ }^{18}$ Sólo en un estudio se evaluó la calidad de vida encontrando mejoras en los pacientes que recibieron IIL, pero no en los pacientes del grupo placebo.

En el estudio de rinoconjuntivitis (RC) se reportó mejoría en la prueba de provocación nasal y en los síntomas locales y sistémicos con la administración de IIL. Sin embargo, no se reportó disminución del uso de medicamentos ni se encontraron cambios significativos en parámetros inmunológicos incluyendo niveles de $\lg \mathrm{E}$, IgG4 séricas o linfocitos CD4+, CD25+, FoxP3+. ${ }^{17}$

Por otra parte, en el estudio de pacientes con rinoconjuntivitis y asma leve (RCyAL), aquellos que recibieron IIL tuvieron mejoras significativas en la prueba de provocación nasal, los síntomas disminuyeron, al igual el uso de medicamentos, y alcanzaron mejoría de los parámetros inmunológicos incluyendo un aumento de $\lg G$ alérgeno específica e lgG4. Sin embargo, a pesar de tener asma bien controlada la prueba de metacolina fue positiva en todos los pacientes que recibieron IIL y no hubo cambios en la calidad de vida (Tabla 2).

Además, en todos los estudios se encontró una adherencia terapéutica apropiada, sólo en un estudio un paciente no completó el tratamiento. ${ }^{15-19}$ 


\section{Eventos adversos}

Al evaluar los eventos adversos ningún estudio reportó reacciones moderadas o graves, sólo se informaron eventos adversos leves como comezón, urticaria, inflamación en sitio de inyección, estornudos y cansancio en las 24 horas posteriores a la inyección. ${ }^{15-19}$

\section{DISCUSIÓN}

En la presente revisión sistemática de ensayos clínicos la finalidad inicial era evaluar la utilidad de la IIL para el tratamiento de enfermedades alérgicas en pacientes pediátricos; sin embargo, al no encontrar ensayos clínicos exclusivamente realizados en pacientes pediátricos se incluyeron estudios que hubiesen incluido a pacientes de edad $\leq 18$ años. En éstos se encontró que la IIL mejoró diversos aspectos clínicos y paraclínicos en pacientes con rinitis alérgica y rinoconjuntivitis. Específicamente se encontraron mejoras en la prueba de provocación nasal, en síntomas locales y sistémicos en todos los estudios evaluados. ${ }^{15-19}$ También la aplicación de la IIL disminuyó el uso de medicamentos e indujo mejoras en parámetros inmunológicos en la mayoría de los estudios (menos en uno de estos). ${ }^{15-19} \mathrm{Si}$ bien la mayoría de los estudios incluidos han sido realizados en pequeños grupos de pacientes, en el total se incluyeron 79 casos y 73 controles y los resultados fueron, en la mayoría de estos, claramente favorables en quienes recibieron IIL en comparación con el placebo, lo que permite determinar que esta terapia es efectiva como se ha postulado de manera individual en los ensayos clínicos previamente realizados. ${ }^{15-19}$

En términos de seguridad, es claro que la IIL es segura, dado que no ocurrieron eventos adversos moderados o graves en los pacientes, sólo eventos adversos leves; au- nado a lo anterior, se reportó una excelente adherencia cercana a $100 \%$, lo cual no se logra con otras formas de inmunoterapia, ya que se ha reportado una adherencia $<70 \%$ a inmunoterapia subcutánea, de $27 \%$ a la inmunoterapia nasal local y de alrededor de $75 \%$ a la inmunoterapia sublingual. ${ }^{20}$ No obstante, vale la pena realizar estudios de mayor tamaño de muestra para estudiar de manera más contundente la adherencia en series mayores de pacientes.

Otros aspectos favorables, además de la eficacia y seguridad de la IIL que se han aclamado en la literatura, es el inicio rápido de acción, los mayores títulos de anticuerpos alcanzados y el menor requerimiento de dosis, lo cual también contribuye a resultados buenos, rápidos y facilita la adherencia. Por lo que, con la evidencia disponible hasta el momento en la literatura, se indica que la IIL es efectiva y segura para el manejo de enfermedades alérgicas como rinitis y rinoconjuntivitis. Falta evidencia para recomendar su uso en asma leve, ya que en el único estudio realizado en pacientes con asma leve no se encontró superioridad de la IIL en comparación con el placebo.

Entre las debilidades de los ensayos clínicos realizados al momento se encuentran: 1. El seguimiento máximo fue de 52 semanas, incluso en un estudio sólo se dio seguimiento por 10 semanas y en dos por 24 semanas; 2 . En un par de estudios el riesgo de sesgo de selección fue alto; 3 . Hace falta un sistema estandarizado de reporte de medición de desenlaces y medición de resultados que podría dar uniformidad, mayor validez y contundencia de ensayos clínicos, y 4. Hace falta la realización de ensayos clínicos en distintas poblaciones a la sueca y norteamericana para dar mayor validez a los resultados.

Además, falta realizar estudios para evaluar la efectividad de la IIL en otras enfermedades alérgicas, y elaborar estudios específicos en pacientes de 15 años o menos, así

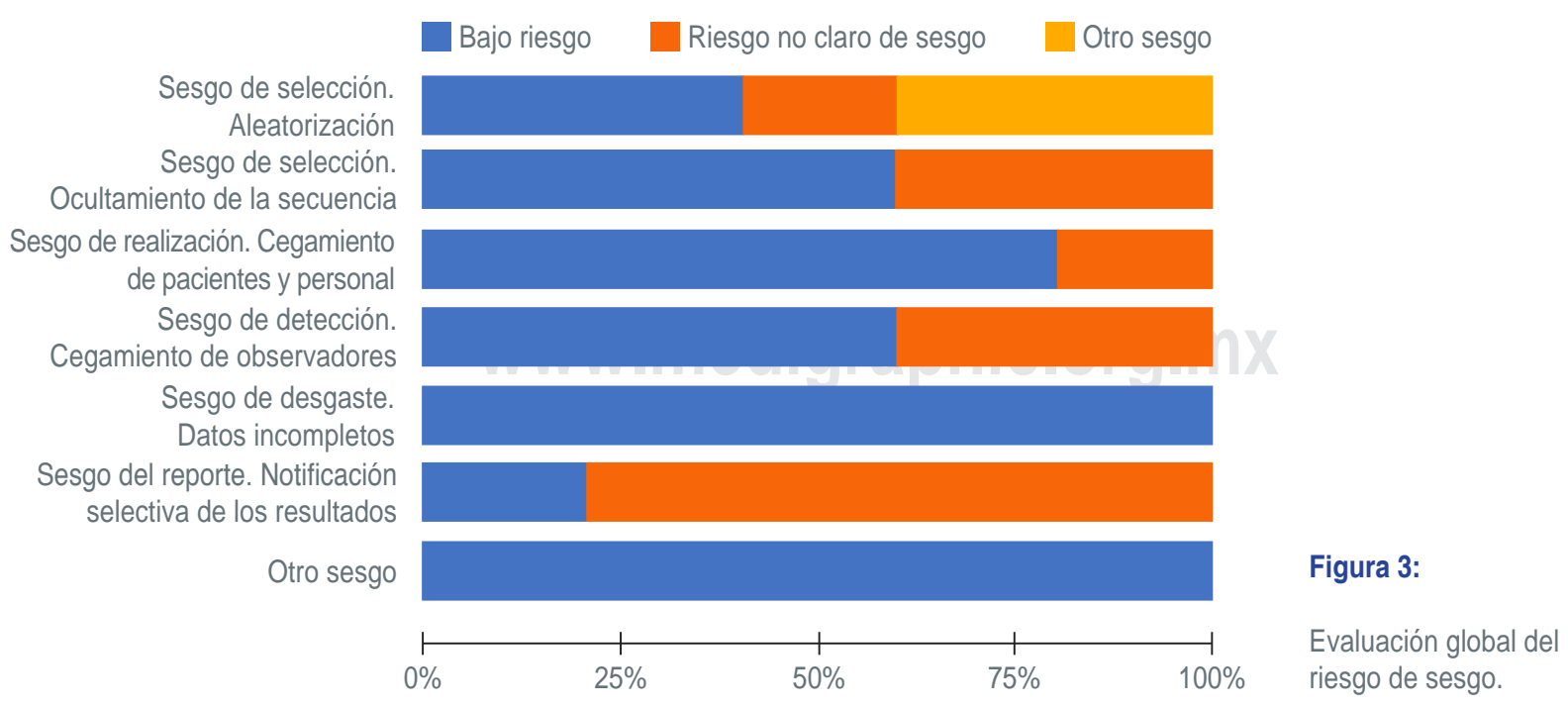


Tabla 2: Resumen de los resultados de la aplicación de la inmunoterapia intralinfática en pacientes con enfermedades alérgicas.

\begin{tabular}{|c|c|c|c|c|c|}
\hline \multirow[b]{2}{*}{ Desenlace medido } & \multicolumn{5}{|c|}{ Estudio } \\
\hline & $\begin{array}{c}\text { Konradsen, } 2020^{19} \\
\text { RCyAL }\end{array}$ & $\begin{array}{c}\text { Hellkvist, } 2018^{18} \\
\text { RA }\end{array}$ & $\begin{array}{c}\text { Hylander, } 2016^{17} \\
\text { RC }\end{array}$ & $\begin{array}{c}\text { Patterson, } 2016^{16} \\
\text { RA }\end{array}$ & $\begin{array}{c}\text { Hylander, } 2013^{15} \\
\text { RA }\end{array}$ \\
\hline $\begin{array}{l}\text { Prueba de } \\
\text { provocación nasal }\end{array}$ & + & + & + & NE & + \\
\hline $\begin{array}{l}\text { Síntomas locales o } \\
\text { sistémicos }\end{array}$ & + & + & + & + & + \\
\hline $\begin{array}{l}\text { Disminución del uso } \\
\text { de medicamentos }\end{array}$ & + & + & SC & + & + \\
\hline $\begin{array}{l}\text { Parámetros } \\
\text { inmunológicos }\end{array}$ & + & + & SC & NE & + \\
\hline Calidad de vida & SC & + & NE & NE & NE \\
\hline
\end{tabular}

$(+)$ = mejoría; $(-)$ = empeoramiento; $\mathrm{SC}=$ sin cambios; $\mathrm{NE}=$ no evaluado; $\mathrm{RCyAL}=$ rinoconjuntivitis y asma leve; $\mathrm{RC}=$ rinoconjuntivitis; $\mathrm{RA}=$ rinitis alérgica.

como determinar cada cuánto tiempo es necesario realizar otras aplicaciones de IIL, ya que los estudios realizados hasta ahora sólo han dado seguimiento máximo de un año.

\section{CONCLUSIONES}

La IIL es una estrategia terapéutica efectiva para el tratamiento de enfermedades alérgicas en pacientes pediátricos y adultos que induce mejorías en las pruebas de provocación nasales, disminuye los síntomas locales y sistémicos, en la mayoría de los casos conduce a la disminución del consumo de medicamentos, mejora parámetros inmunológicos y puede mejorar la calidad de vida. Además, se asocia con buena adherencia terapéutica y eventos adversos leves, pero no moderados ni severos.

\section{REFERENCIAS}

1. Incorvaia C, Ciprandi G, Nizi MC, Makri E, Ridolo E. Subcutaneous and sublingual allergen-specific immunotherapy: a tale of two routes. Eur Ann Allergy Clin Immunol. 2020; 52 (6): 245-257.

2. Naeraa SH, Schollert NH, Flader Skov PN, Homøe P. Intralymphatic allergen-specific immunotherapy. Ugeskr Laeger. 2018; 180 (22): V09170695.

3. Akdis CA, Akdis M. Mechanisms of allergen-specific immunotherapy. J Allergy Clin Immunol. 2011; 127 (1): 18-19. doi: 10.1016/j. jaci.2010.11.030.

4. Głobinska A, Boonpiyathad T, Satitsuksanoa P, Kleuskens M, van de Veen W, Sokolowska M et al. Mechanisms of allergen-specific immunotherapy: Diverse mechanisms of immune tolerance to allergens. Ann Allergy Asthma Immunol. 2018; 121 (3): 306-312. doi: 10.1016/j.anai.2018.06.026.

5. Senti G, Prinz Vavricka BM, Erdmann I, Diaz MI, Markus R, McCormack SJ et al. Intralymphatic allergen administration renders specific immunotherapy faster and safer: a randomized controlled trial. Proc Natl Acad Sci U S A. 2008; 105 (46): 17908-17912. doi: 10.1073/pnas.0803725105.
6. Ravi A, Rank MA. Reducing and managing systemic reactions to immunotherapy. Curr Opin Allergy Clin Immunol. 2013; 13 (6): 651-655. doi: 10.1097/ACI.0b013e328364c876.

7. van Zelm MC, McKenzie Cl, Varese N, Rolland JM, O'Hehir RE. Recent developments and highlights in immune monitoring of allergen immunotherapy. Allergy. 2019; 74 (12): 2342-2354. doi: 10.1111/all.14078.

8. Senti G, Kündig TM. Intralymphatic immunotherapy. World Allergy Organ J. 2015; 8 (1): 9. doi: 10.1186/s40413-014-0047-7.

9. Martínez-Gómez JM, Johansen P, Erdmann I, Senti G, Crameri R, Kündig TM. Intralymphatic injections as a new administration route for allergen-specific immunotherapy. Int Arch Allergy Immunol. 2009; 150 (1): 59-65. doi: 10.1159/000210381.

10. Cox L, Nelson H, Lockey R et al. Allergen immunotherapy: a practice parameter third update. J Allergy Clin Immunol. 2011; 127 (1 Suppl): S1-55. doi: 10.1016/j.jaci.2010.09.034.

11. Senti G, Freiburghaus AU, Larenas-Linnemann D et al. Intralymphatic Immunotherapy: update and unmet needs. Int Arch Allergy Immunol. 2019; 178 (2): 141-149. doi: 10.1159/000493647.

12. Stovold E, Beecher D, Foxlee R, Noel-Storr A. Study flow diagrams in Cochrane systematic review updates: an adapted PRISMA flow diagram. Syst Rev. 2014; 3: 54. doi: 10.1186/2046-4053-3-54.

13. Moher D, Shamseer L, Clarke M, Ghersi D, Liberati A, Petticrew $M$ et al. Preferred reporting items for systematic review and metaanalysis protocols (PRISMA-P) 2015 statement. Syst Rev. 2015; 4 (1): 1.

14. Higgins JP, Altman DG, Gotzsche PC, Juni P, Moher D, Oxman AD et al. The Cochrane Collaboration's tool for assessing risk of bias in randomised trials. BMJ. 2011; 343: d5928. doi: 10.1136/bmj. d5928.

15. Hylander T, Latif L, Petersson-Westin U, Cardell LO. Intralymphatic allergen-specific immunotherapy: an effective and safe alternative treatment route for pollen-induced allergic rhinitis. J Allergy Clin Immunol. 2013; 131 (2): 412-420. doi: 10.1016/j. jaci.2012.10.056.

16. Patterson AM, Bonny AE, Shiels WE 2nd, Erwin EA. Three-injection intralymphatic immunotherapy in adolescents and young adults with grass pollen rhinoconjunctivitis. Ann Allergy Asthma Immunol. 2016; 116 (2): 168-170. doi: 10.1016/j.anai.2015.11.010.

17. Hylander T, Larsson O, Petersson-Westin U, Eriksson M, Kumlien Georén S, Winqvist $O$ et al. Intralymphatic immunotherapy of pollen-induced rhinoconjunctivitis: a double-blind placebo-controlled trial. Respir Res. 2016; 17: 10. doi: 10.1186/s12931-016-0324-9. 
18. Hellkvist L, Hjalmarsson E, Kumlien Georén S, Karlsson A, Lundkvist K, Winqvist $\mathrm{O}$ et al. Intralymphatic immunotherapy with 2 concomitant allergens, birch and grass: A randomized, double-blind, placebo-controlled trial. J Allergy Clin Immunol. 2018; 142 (4): 1338-1341.e9.

19. Konradsen JR, Grundström J, Hellkvist L et al. Intralymphatic immunotherapy in pollen allergic young adults with rhinoconjunctivitis and mild asthma: a randomized trial. J Allergy Clin Immunol. 2020; 145 (3): 1005-1007. doi: 10.1016/j.jaci.2019.11.017.

20. Senna G, Ridolo E, Calderon M, Lombardi C, Canonica GW, Passalacqua G. Evidence of adherence to allergen-specific immunotherapy. Curr Opin Allergy Clin Immunol. 2009; 9 (6): 544-548. doi: 10.1097/ACl.0b013e328332b8df. 